\title{
A Comparative Evaluation of Timolol Maleate and Pilocarpine in the Treatment of Chronic Open Angle Glaucoma
}

\author{
N. L. DALLAS, W. E. SPONSEL and A. J. HOBLEY \\ Bristol
}

\begin{abstract}
Summary
Ninety-two eyes with newly-diagnosed chronic open angle glaucoma (COAG) were treated in a randomised prospective trial with either timolol or pilocarpine. Their visual field survival was monitored on a 3-monthly basis over 2 years using both Goldmann and Friedmann perimetry. Concomitant tonometric data was derived by applanation.

Fields were assessed and quantified using algorithms designed to give the greatest sensitivity for glaucomatous field loss. Microcomputer programmes specifically designed for this purpose were used in the data collection and subsequent analysis.
\end{abstract}

Comparative studies showing that competing forms of therapy can have a different influence in the sparing of visual fields, would provide the best means of establishing the fact that current practice does yield substantial 'benefit'. Unfortunately the majority of studies have concentrated on intra ocular pressure effects without addressing themselves to the question of field preservation.

Only by accurate estimation of neural function can therapeutic efficacy be demonstrated conclusively. Hence, the present study was carried out in order to obtain an objective evaluation of visual field survival, the definitive criterion of therapeutic success or failure in glaucoma management. The methods used for field evaluation were systematically derived, highly accurate and fully reproducible. The scoring systems used yielded patient data of a consistency sufficient to provide significant field trends for a substantial proportion of the eyes under study. More extensive accounts of the scoring technique and this trial data have been published elsewhere. ${ }^{1,2}$

\section{Material and Methods}

Patients:

A total of 50 patients, 25 in each group, were selected on a progressive, randomised entry basis, provided they met the following criteria:

(i) IOP $>21$ on two occasions

(2) optic disc cupping supportive of a diagnosis of glaucoma

(3) visual field loss typical of nerve fibre bundle damage caused by glaucoma.

Patients were excluded if they had:

(1) contra-indications as shown on the Timoptol data sheet

(2) visual ability $6 / 9$ or worse

(3) retinal problems likely to affect field plotting

or if it was thought they might prove to be unreliable

Treatment and Dose Adjustment:

(a) Timoptol group: $0.25 \%$ b.d. for 2 weeks. If IOP was still above $22 \mathrm{~mm}$. Hg. then treatment was increased to Timoptol $0.5 \%$ b.d.

(b) Pilocarpine group: $2 \%$ b.d. for 2 
weeks. If IOP remained above $22 \mathrm{~mm} . \mathrm{Hg}$. treatment was increased to Pilocarpine $4 \%$ b.d

\section{Follow-up:}

Patients were followed at 3-monthly inter vals for two years. IOP and perimetry were recorded at each attendance. Field charts were quantified on the Apple computer and a linear plot drawn. If a deteriorating trend in the visual field was thought to be highly significant, the patient was withdrawn from the trial. An adverse reaction to treatment also necessitated the patient's withdrawal from the study.

Analysis:

This was directed towards

(1) duration of IOP control.

(2) visual field maintenance or change

(3) patient outcome, e.g. number requiring additional therapy or trabeculectomy. Attrition from trial:

Twenty-one eyes of 13 patients.

(a) 8 eyes of 8 patients were on

Pilocarpine

(b) 2 eyes of 1 patient were on

Timoptol

(c) 2 patients were lost to follow-up

(d) 8 eyes were submitted to trabecular surgery (4 from each group)

Sex Distribution:

This was well matched $60 \%$ males, $40 \%$ females

Age Distribution:

The mean age of the Timoptol group was 62.5 years and mean age of Pilocarpine group was 68.7 years

Age Distribution according to sex:

There was a slight seniority (2-3 years) of male patients.

Methods

(a) Tonometry was carried out by Goldmann applanation

(b) Goldmann perimetry was performed on isopters $\mathrm{I} 2 \mathrm{e}, \mathrm{I} 3 \mathrm{e}, \mathrm{I} 4 \mathrm{e}$ and III $4 \mathrm{e}$ and the score system displayed as Goldmann radial units (GRU), a method described in a previous paper. ${ }^{3}$

(c) Friedmann suprathreshold static perimetry was carried out routinely and scored by a method devised by Henson ${ }^{4}$ (University of Wales Institute of Science and Technology). This is a system which takes account of position, depth and clustering of 'missed points', and the computer-assisted conversions give easy-toread two dimensional data.

(d) The micro computer system has also been decribed previously. ${ }^{5}$ The cumulative data analysis is available on two separate print outs showing the field analysis graphically and a score sheet giving linnear progression of IOP readings, GRUs and Friedmann scores.

\section{Results}

Figure 1 shows the distribution of initial intraocular pressures and Friedmann scores for patients in both treatment groups. It can be seen that no correlation exists between intraocular pressures and initial field scores; indeed, the worst visual function was found in those with lower intraocular pressure levels at presentation. It is also noteworthy that those individuals with high pressures and minimal field deficit were not "ocular hypertensives", but had indeed presented with fundal characteristics consistent with a clinical diagnosis of COAG. The truncation of this distribution of pressures below $20 \mathrm{~mm}$. $\mathrm{Hg}$. should not be construed to represent a true physiological cut-off point, but is merely a manifestation of the entry requirements for the study. Certainly it would appear highly probable that assessment of referrals with lower pressures would yield many further cases of significant visual field deficit.

\section{Friedmann Field Responses over 18 months}

Comparative assessment of the progress of patients on the two drugs was performed by considering the mean Friedmann field score change from the mean in initial field score value for each patient group, at threemonthly intervals.

The analysis suggests an association between a transient period of Friedmann field score improvement and timolol therapy (Fig. 2). This effect appeared to develop during the first three months of therapy, and was sustained in the timolol-treated population over the first year of treatment.

The Pilocarpine-treated group, however, showed an immediate and sustained linear 


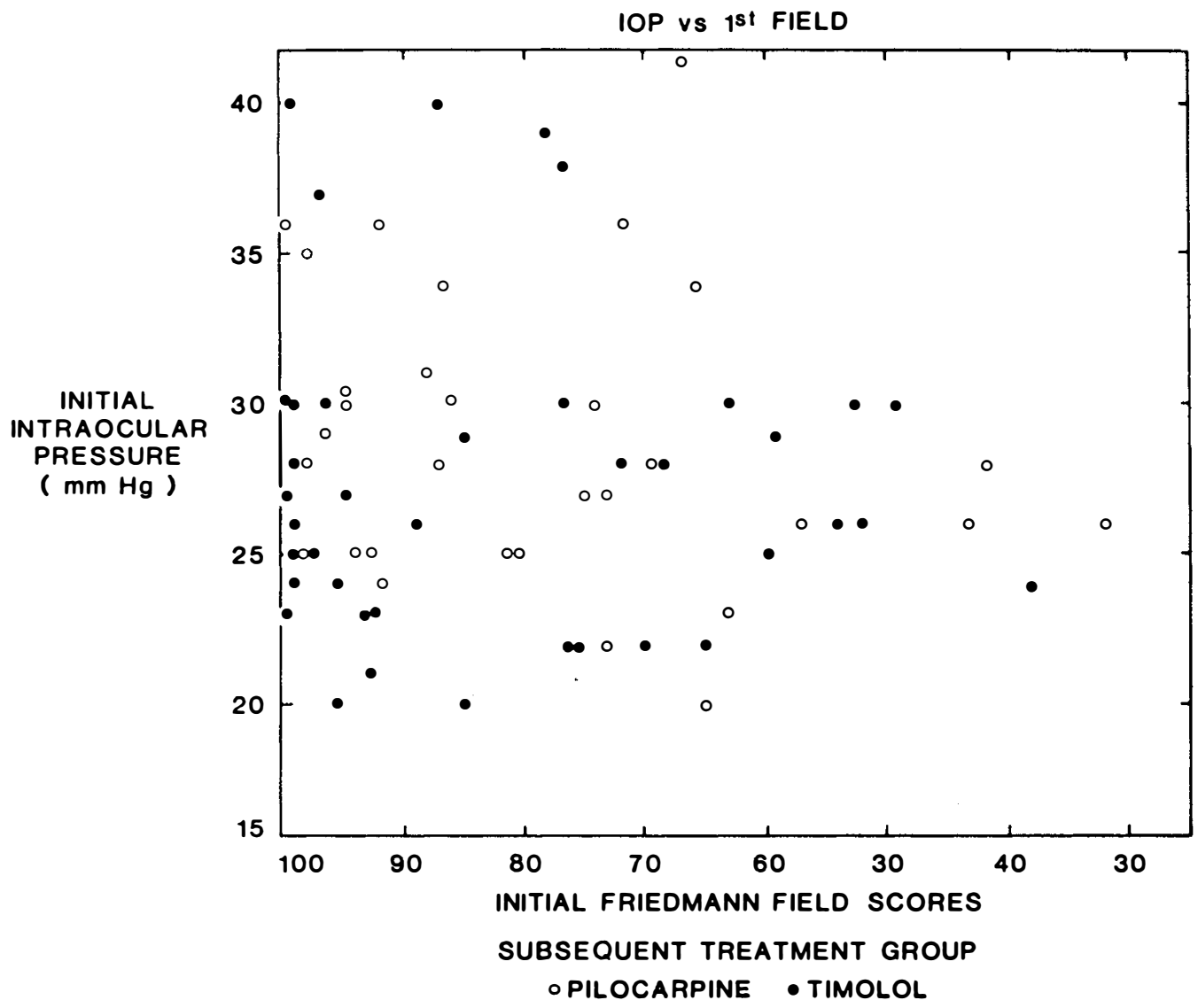

Fig. 1. The distribution of initial intraocular pressures and Friedmann scores for patients in both treatment groups.

downward progression in Friedmann field scores.

A breakdown of the individual figures for field scores after the initial recovery phase shows that progressive field loss occurred later in $24 \%$ of timolol-treated eyes.

Fifty-nine per cent showed no significant rate of change, and three eyes on timolol $(9 \%)$ showed ongoing trends of significant central field improvement.

Comparative assessment of the progress of patients on the two drugs was performed by considering the mean Goldmann score change between the first visit and those obtained at subsequent visits within threemonthly intervals, beginning at 4 months. There was no significant difference in the 3monthly field survival responses of the two patient groups at isopter $12 \mathrm{e}$ on the basis of this analysis.

The overall difference between the field survival scores of the two patient groups at isopter $14 \mathrm{e}$ also failed to reach a conventional level of significance. A consideration of IOP reduction in these groups in terms of mean percentages of pressure reduction yields no useful correlation with field survival response. Some eyes showing severe deterioration had actually undergone a significantly greater degree of IOP reduction than those exhibiting progressive field improvement!

There were no effective therapeutic differences between these two groups. All 34 eyes showing significant field trends, positive or negative, responded to therapy with reduction in their mean IOP values. 


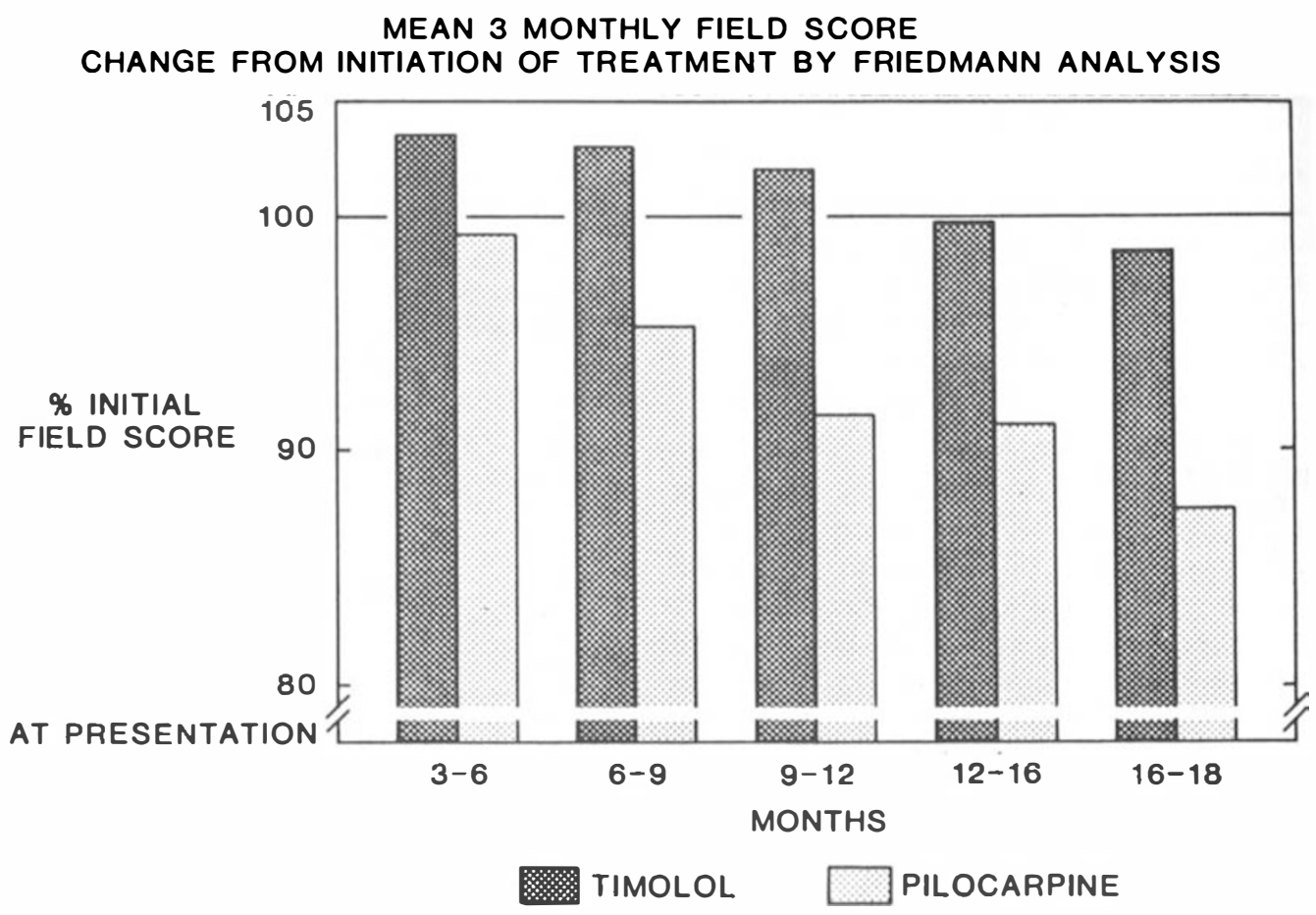

Fig. 2. Friedmann score change with the two drugs at 3-month intervals (relative to mean group scores at presentation). Note the positive effect observed for the timolol-treated population, sustained over the first year of therapy.

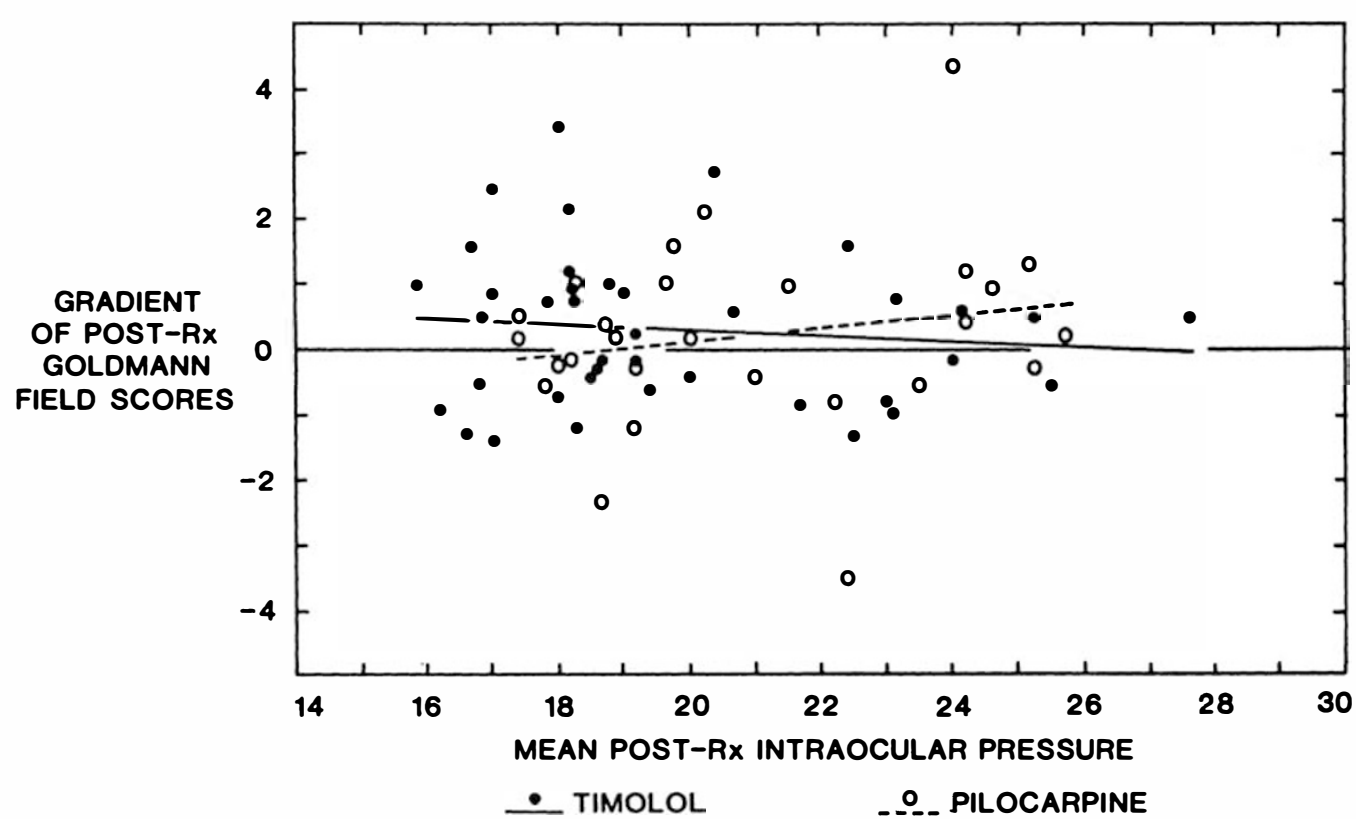

Fig. 3. Relationship between post-Rx IOP and field score gradients as calculated from I2e Goldmann perimetric traces. A similar lack of correlation is observed at all other isopters tested. 
Relationship of Initial Field Score to Subsequent Field Survival Trend:

One widely asserted opinion is that patients presenting with a more severe degree of field loss initially will tend to progress more rapidly than those detected at an earlier stage. There was clearly no significant correlation between these two variables in the patient population tested.

Figure 3 shows a relationship between post- treatment IOP and field score gradients as calculated from $12 \mathrm{e}$ Goldmann perimetric traces.

\section{Discussion}

Large scale epidemiological studies in the United States of America and elsewhere have repeatedly shown a low association between raised IOP and glaucomatous visual field loss. ${ }^{6,7}$ An interpretation of the results of these studies suggests that an examination of all individuals with mean IOPs over 21 $\mathrm{mm}$. $\mathrm{Hg}$. would result in a failure to detect over $60 \%$ of glaucomatous defects; that is,

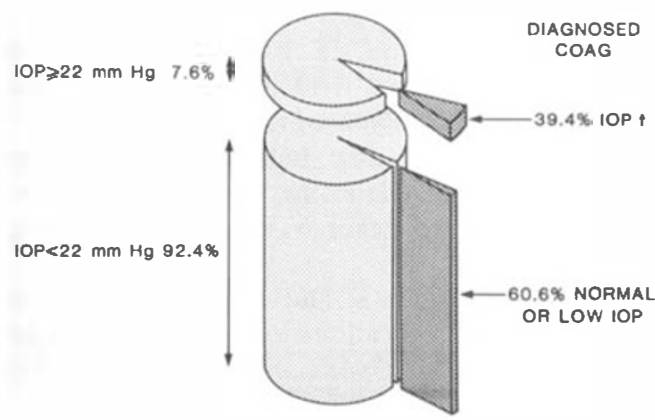

Fig. 4. The sensitivity/specificity relationship between intraocular pressure and glaucomatous field loss. Adapted from Leibowitz $z^{6}$ pressure and glaucomatous field loss. Adapted from Leibowitz Hollows and Graham ${ }^{7}$ \& Henson ${ }^{13}$, from the Framington Study 1980. This figure demonstrates that in a comprehensive sampling of the population at large using Armaly-Drance modified kinetic perimetry, over $60 \%$ of glaucomatous fields were found amongst individuals having normotensive or even hypotensive IOP levels. Conversely, fewer than one in twelve persons exhibiting ocular hypertension showed evidence of field loss by the same criteria. These epidemiological data confirm both the low sensitivity and low specificity of IOP as an indicator of glaucomatous neural degeneration. missing 3 out of 5 affected individuals (Fig. 4 ). Neither was there any correlation, in our series, between initial IOP presentation and the severity of central field loss.

One also finds that in analysis of the progress of glaucoma under various forms of therapy no useful correlation has been established between the measured IOP reductions achieved by medical or surgical therapy and any ensuing visual field change..$^{8-13}$ All of the usual forms of therapy provided sustained reductions in the IOP of patients who had previously had abnormally high pressures. In each case correlation between the extent of IOP reduction and field survival was minimal or absent. Some forms of therapy may be better than others in terms of field survival. These facts justify a search for therapeutic agents whose beneficial effects may be entirely IOP-independent.

The weight of evidence against there being a direct cause-and-effect relationship between intraocular hypertension and $\mathrm{COAG}$ is strong. In the $\mathrm{KCH}$ data study the figures show that if all eyes with ocular hypertension were treated successfully and removed from the data, the vast majority of those with glaucomatous field loss would remain. ${ }^{14}$

Although excessively high, IOP itself may not be the primary cause of COAG. A disturbed aqueous circulation might nevertheless remain a likely influence in the cause of glaucoma field loss, and most present treatments are designed to restore or modulate the aqueous circulation. Clearly, however, simple measurement of IOP in a clinical setting has very limited value in detecting or monitoring eyes with COAG as so many factors influence the intraocular pressure.

Even after trabeculectomy (or other drainage procedure) there is a poor correlation between IOP reduction and field survival. ${ }^{13}$

If one is looking for other causes of nerve destruction in COAG attention should be paid to the optic nerve where vascular insufficiency is important. Our observation of an IOP-independent restorative field effect of timolol may be related to the drug's ability to selectively enhance choroidal and retinal circulation. ${ }^{15,16}$ In the process of disc cupping there is loss of glial tissue as well as nerve 
fibres, and a chronic ischaemic basis for cupping seems more plausible than direct hydrostatic pressure. The glial/neural shrinkage may exacerbate vascular insufficiency and/or inhibit axoplasmic transport from ganglion cells to the lateral geniculate bodies.

Refinements in perimetry are important and we believe that improved methods of data analysis are even more so.

\section{Conclusion}

In the study the perimetric reproducibility was very high, and the transcription of field charts into computer memory precise.

The findings reported here fully corroborate those reported in our earlier retrospective study which concluded that field survival provides a far more consistent guide to the progress of glaucoma under treatment than does IOP $(\mathrm{p}<<0.001)$. The apparent lack of any useful correlation between IOP and visual field survival in many eyes was clearly noted at that time. ${ }^{3}$

The results of this trial suggest that, within the observed IOP limits, intraocular pressure measurements provide less useful information to the clinician concerning the visual fate of the patients once they have commenced treatment with pilocarpine or timolol.

The most immediately striking finding in terms of field response to treatment is the significant differences observed between the timolol and pilocarpine groups as compared by Friedmann central field analysis. Improvement in Friedmann scores developed over the course of the first three months. The basis for this marginal improvement in the timolol group is uncertain.

Conversely, the pilocarpine-treated eyes showed an immediate and sustained downward progression of central field function and the functional level in the timolol-treated patients is superior to that in the pilocarpinetreated group at every stage examined. Timolol seems to 'buy time' in addition to giving the transient improvement in some eyes.

The pressure lowering effects of the two drugs in this trial were virtually identical, but one drug appears to have significantly improved central field function, while the other has given negative field response.

\section{References}

1 Sponsel WE, Hobley AJ, Dallas NL, Henson DB. Visual field survival; a comparison of the effects of pilocarpine and timolol in open angle glaucoma. Proc XXV Int Cong Ophthal Glaucoma Society. In; New Trends in Ophthalmology (CIC, Roma) 1986; In Press.

2 Sponsel WE, Hobley, AJ, Williams AH, Dallas NL. Glaucoma assessment by microcomuter. Trans Ophthalmol Soc UK 1984; 104: 100-5.

3 Sponsel WE, Dallas NL, Burbridge L. Visual field survival: the response to timolol therapy in open-angle glaucoma. $\mathrm{Br} \mathrm{J}$ Ophthalmol 1983; 67: 220-7.

${ }^{4}$ Henson DB, Dix SM. Evaluation of the Friedman Visual Field Analyser Mark II. Part 2. Results from a population with induced visual field defects. Br J Ophthalmol 1984; 68: 463-7.

${ }^{5}$ Sponsel WE, Williams AH, Dallas NL, Henson DB. A microcomputer-based glaucoma assessment system for clinical ophthalmologists. Res and Clin Forums 1983; 5: 25 35.

${ }^{6}$ Leibowitz HM et al. The Framingham Study Monograph, Section IV. Glaucoma. Surv Ophthalmol 1980; 24: 336-76.

${ }^{7}$ Hollows FC, Graham PA. Intraocular pressure, glaucoma, and glaucoma suspects in a defined population. Br J Ophthalmol 1966; 50: 570-86.

${ }^{8}$ Holmin C, Krakau CET. Regression analysis of the central visual field in chronic glaucoma cases. Acta Ophthalmol 1982; 60: 267-74.

9 Krakau CET. Visual field decay in chronic glaucoma. In Glaucoma Update II (Krieglstein GK, Leydhecker W, eds) Springer Verlag 1983; 123-8.

${ }^{10}$ Flammer J, Drance S. The effect of a number of glaucoma medications on differential light threshold. Doc Ophthalmol Proc Series 1983; 35: 145-55.

${ }^{11}$ Helman K. Progression and regression of visual field defects. In Glaucoma: Conceptions of a Disease (Heilman K, Richardson KT, eds) George Theime Pub, Stuttgart, 1978; 168-75.

12 Heijl A, Bengtsson B. Does laser-induced reduction of intraocular pressure improve the glaucomatous visual field? Acta Ophthalmol 1984; 62: 705-14.

13 Kidd MA, O'Conner M. Progression of field loss after trabeculectomy: a five-year followup. Br J Ophthalmol 1985; 69: 827-31.

14 Daubs J, Crick RP. Epidemiological analysis of King's College Hospital glaucoma data. Res \& Clin Forums 1980; 2: 41-59.

15 Ernest JT, Goldstick TK. Timolol maleate and choroidal blood flow. Glaucoma Update II 
(Krieglstein GK, Leydhecker W, eds) Springer Verlag 1983; 45-50.

${ }^{16}$ Grunwald J. Laser doppler velocimetric study of the effect of topical timolol on the normal human retinal circulation. Proc XXV Int
Cong Ophthalmol Glaucoma Society, In New Trends in Ophthalmology (CIC, Roma) 1986; (In Press).

17 Henson DB. Intraocular pressure n glaucoma screening. The Optician, Sept 14, 1984; 10-12. 